\title{
Synergistic Inhibition of Renal Fibrosis by Nintedanib and Gefitinib in a Murine Model of Obstructive Nephropathy
}

\author{
Liu Feng ${ }^{a}$ Wang Lia Yu Chao ${ }^{a}$ Qin Huan ${ }^{a}$ Fang Lu ${ }^{a}$ Wang Yia Wang Jun ${ }^{a}$ \\ Cui Binbin ${ }^{a}$ Liu Na ${ }^{a}$ Zhuang Shouganga, b \\ ${ }^{a}$ Department of Nephrology, Shanghai East Hospital, Tongji University School of Medicine, Shanghai, China; \\ ${ }^{b}$ Department of Medicine, Rhode Island Hospital and Alpert Medical School, Brown University, Providence, RI, USA
}

\section{Keywords}

Nintedanib - Gefitinib · Receptor tyrosine kinases · Renal

fibrosis · Unilateral ureteral obstruction

\begin{abstract}
Background: Our recent studies demonstrated that both nintedanib, an FDA-approved quadruple kinase inhibitor, and gefitinib, an epidermal growth factor receptor (EGFR) inhibitor, protect against obstructive kidney disease. It remains unknown whether they have a synergistic effect. Methods: In this study, we investigated the effect of combined administration of nintedanib and gefitinib on renal fibrosis in a murine model of renal fibrosis induced by unilateral ureteral obstruction (UUO). Results: Combined treatment with nintedanib and gefitinib after UUO resulted in a greater antifibrotic effect compared with their individual application. Mechanistically, administration of nintedanib blocked UUOinduced phosphorylation of multiple kinase receptors associated renal fibrosis, including platelet-derived growth factor receptors, fibroblast growth factor receptors, vascular endothelial growth factor receptors, and Src family kinase, while gefitinib inhibited EGFR phosphorylation. Their combination also exhibited a more pronounced effect in reducing expression of tissue inhibitors of metalloproteinase-2 (TIMP-2), increasing expression of matrix metalloproteinase-2 (MMP-2), and suppressing renal proinflammatory cytokine expression
\end{abstract}

and macrophage infiltration in the injured kidney. Furthermore, simultaneous administration of nintedanib and gefitinib was more potent in inhibiting UUO-induced renal phosphorylation of signal transducer and activator of transcription-3 (STAT3), nuclear factor-KB, and Smad-3 compared with monotherapy. In cultured renal interstitial fibroblasts, cotreatment with these 2 inhibitors also had synergistic effects in abrogating transforming growth factor $\beta 1$-induced activation of renal fibroblasts and phosphorylation of Akt, STAT3, and Smad3. Conclusions: Combined application of nintedanib and gefitinib has a synergistic antifibrotic effect in the kidney and may hold translational potential for the treatment of chronic kidney disease.

(c) 2020 The Author(s)

Published by S. Karger AG, Basel

\section{Introduction}

Renal fibrosis is characterized by activation of renal fibroblasts and deposition of extracellular matrix (ECM) proteins [1] and the final common pathway in the progression of chronic kidney disease (CKD) to end-stage renal disease (ESRD) [2]. Although many animal studies have revealed the pathogenesis and potential therapeutic targets of renal fibrosis, only very limited basic findings have been translated into clinical application to prevent renal fibrosis development or slow CKD progression [3]. karger@karger.com www.karger.com/kdd

Karger $\stackrel{\text { ' }}{5}$

GOPEN ACCESS
(C) 2020 The Author(s)

Published by S. Karger AG, Basel

This article is licensed under the Creative Commons AttributionNonCommercial-NoDerivatives 4.0 International License (CC BYNC-ND) (http://www.karger.com/Services/OpenAccessLicense). Usage and distribution for commercial purposes as well as any distribution of modified material requires written permission.
Zhuang Shougang

Department of Nephrology, Shanghai East Hospital

Tongji University School of Medicine, 1800 Yuntai Road

Shanghai 200123 (China)

szhuang@lifespan.org 
As such, searching for effective antifibrotic agents with the best safety profile is still a major challenge [2].

Our studies and others have demonstrated that receptor tyrosine kinases (RTKs) and nonreceptor tyrosine kinases (nRTKs) are involved in the initiation and progression of renal fibrosis [4]. RTKs are membrane receptors, classified into a variety of superfamilies, including platelet-derived growth factor receptors (PDGFR), vascular endothelial growth factor receptors (VEGFR), fibroblast growth factor receptors (FGFR), and epidermal growth factor receptors (EGFR). nRTKs such as Src, c-Abl, and c-kit, lack extracellular and transmembrane domains and mediate activation of some intracellular signaling pathways [4]. Activation of RTKs and Src can induce epithelial-mesenchymal transition (EMT) of renal tubular cells, inflammatory cell infiltration, and ECM accumulation and metabolism [4]. Given that multiple RTKs and Src play roles in the pathogenesis of renal fibrosis, targeting more than one RTK and nRTKs might result in a better antifibrotic effect [4].

Nintedanib is a multiple-target tyrosine kinase inhibitor (TKI) that can inhibit phosphorylation of PDGFR, VEGFR, FGFR, and Src family kinases. It has been shown that nintedanib exhibits a powerful antifibrotic effect in idiopathic pulmonary fibrosis (IPF) [5], liver fibrosis [6], and systemic sclerosis $[7,8]$. Administration of nintedanib also effectively improved lung function in patients with IPF $[9,10]$, for which it was designated a breakthrough treatment therapy [11]. Our recent studies demonstrated that nintedanib could also abrogate renal fibrosis and partially reversed established renal fibrosis through the mechanisms associated with blockade of multiple RTKs, Src family kinases, and TGF- $\beta$ signaling [12]. In addition, we showed that gefitinib, a selected inhibitor of EGFR, exhibited an antifibrotic effect in murine models of renal fibrosis and peritoneal fibrosis $[13,14]$. Given that development of renal fibrosis is mediated by multiple RTKs and nRTKs, it is assumed that combined application of nintedanib and gefitinib would provide a synergistic antifibrotic effect in the kidney. The purpose of the present study was to assess the possible synergistic antifibrotic effects of nintedanib and gefitinib in a murine model of renal fibrosis induced by UUO and in cultured renal fibroblasts.

\section{Materials and Methods}

Chemicals and Antibodies

Antibodies to p-PDGFR- $\beta$, p-VEGFR2, p-STAT3, STAT3, pEGFR, EGFR, p-Smad3, Smad3, p-NF-kBp65, p-Akt, Akt, and $\beta$-actin were purchased from Cell Signaling Technology (Danvers,
MA, USA). TGF- $\beta 1$ and antibodies to type I collagen and fibronectin were purchased from Santa Cruz Biotechnology (Santa Cruz, CA, USA). p-FGFR1 antibody was purchased from Lifespan Biosciences (Seattle, WA, USA). NF- $\kappa$ Bp65 antibody was purchased from ProSci Inc (San Diego, CA, USA). CD68 antibodies and MCP-1, TNF- $\alpha$, IL-1 $\beta$, and IL-6 ELISA assay kits were purchased from Abcam Inc (Cambridge, UK). Nintedanib was purchased from Cayman (Arbor, MI, USA). F4/80 antibody was purchased from Servicebio Inc (Wuhan, China). Gefitinib was purchased from LC Laboratories (Woburn, MA, USA). a-SMA antibody and all other chemicals were purchased from Sigma (St. Louis, MO, USA).

\section{Mouse Models of Renal Fibrosis and Nintedanib and Gefitinib} Administration

The UUO model was established in male C57/black mice weighing 20-25 g with an average age of 6-8 weeks (Shanghai Super-B\&K Laboratory Animal Corp. Ltd., Shanghai, China) as described previously [12]. In brief, the abdominal cavity of the animal was exposed via a midline incision, and the left ureter was isolated and ligated. Sham-operated animals received the same surgical procedures but without ureter ligation. Forty-eight male mice were randomly assigned to 8 groups of 6 mice each: sham + DMSO, sham treated with nintedanib, sham treated with gefitinib, and sham treated with both nintedanib and gefitinib; UUO + DMSO, UUO treated with nintedanib, UUO treated with gefitinib, and UUO treated with both nintedanib and gefitinib. To examine the effect of nintedanib and/or gefitinib on the development of renal fibrosis, nintedanib at $50 \mathrm{mg} / \mathrm{kg}$ was given by gavage and gefitinib at $100 \mathrm{mg} / \mathrm{kg}$ by peritoneal injection immediately after ureteral ligation and then administered daily. DMSO alone treated animals were used as controls. At day 7 after surgery, kidneys were harvested. All animal studies were performed according to the US Guidelines on the Care and Use of Laboratory Animals and approved by the Tongji University Animal Welfare Committee.

\section{Cell Culture and Treatments}

Normal rat kidney fibroblast (NRK-49F) cells were cultured in Dulbecco's modified Eagle's medium (DMEM; Sigma-Aldrich, St. Louis, MO, USA) containing $5 \%$ fetal bovine serum (FBS), $0.5 \%$ penicillin, and streptomycin in an atmosphere of $5 \% \mathrm{CO}_{2}$ and $95 \%$ air at $37^{\circ} \mathrm{C}$. To determine the effects of nintedanib and/or gefitinib treatment on fibroblast activation, NRK-49F cells were starved for $24 \mathrm{~h}$ by incubation with DMEM containing $0.5 \%$ FBS followed by stimulation with TGF- $\beta 1(5 \mathrm{ng} / \mathrm{mL})$ for $24 \mathrm{~h}$ and then incubated with $400-$ nM nintedanib and/or 10-nM gefitinib for an additional $48 \mathrm{~h}$.

\section{Immunoblot Analysis}

Immunoblot analysis of tissue samples and NRK- $49 \mathrm{~F}$ cells was conducted as described previously [12]. The densitometry analysis of immunoblot results was conducted using ImageJ software developed at the US National Institute of Health. The quantification data are given as ratio between the target protein and loading control.

\section{Histochemical, Immunohistochemical, and}

Immunofluorescence Analysis

Formalin-fixed kidneys were embedded in paraffin and prepared in $3-\mu \mathrm{m}$-thick sections. Immunohistochemical staining was conducted on the basis of the procedure described in our previous study [12]. Hematoxylin and eosin (HE) staining was performed on the renal tissue specimens to observe the morphological changes of 
the renal tissue and cells. To evaluate renal fibrosis, Masson trichrome staining was performed according to the protocol provided by the manufacturer (Sigma-Aldrich, St. Louis, MO, USA). The collagen tissue area (green/blue color) was quantitatively measured using Image Pro-Plus software (Media Cybernetics, Silver Spring, MD, USA) by drawing a line around the perimeter of positive staining area, and the average ratio of collagen tissue area to total area in each microscopic field $(\times 200)$ was calculated and graphed. Infiltration of $\mathrm{CD} 68^{+}$and $\mathrm{F} 4 / 80^{+}$macrophages in kidney tissue was assessed by immunohistochemical staining. F4/80 expression in kidney tissue was assessed by immunofluorescent staining using a fluorescence confocal microscope (NIKON, Japan).

\section{ELISA Analysis}

To examine renal expression of MCP-1, TNF- $\alpha$, IL- $1 \beta$, and IL6 , murine kidneys were homogenized in an extraction buffer. The supernatant recovered after centrifugation was used to determine these chemokines/cytokines by using the commercial Quantikine ELISA kits in accordance with the protocol specified by the manufacturer (Abcam Inc, Cambridge, UK). Total protein levels were determined using a bicinchoninic acid protein assay kit. The concentration of cytokines in kidneys was expressed as picograms per milligram of total proteins.

\section{Statistical Analysis}

All the experiments were conducted at least 3 times. Data depicted in graphs represent the means \pm SEM for each group. Intergroup comparisons were made using one-way analysis of variance (ANOVA). Multiple means were compared using Tukey's test. The differences between 2 groups were determined by Student's $t$ test. Statistically significant differences between mean values were marked in each graph. $p<0.05$ was considered a statistically significant difference between mean values. All the statistical analyses were conducted by SPSS 20.0 .

\section{Results}

Synergistic Inhibition of Renal Fibrosis by

Combined Administration of Nintedanib and

Gefitinib in a Murine Model of UUO

Our previous studies have shown that either nintedanib or gefitinib attenuates renal fibrosis [12]. To understand whether these 2 agents have a synergistic effect in inhibiting renal fibrosis, we first examined the efficacy of nintedanib or gefitinib given alone or their combination on the changes in collagen fibers by Masson staining in the kidney after UUO injury. Gefitinib was administered at $100 \mathrm{mg} / \mathrm{kg}$ by peritoneal injection and nintedanib at 50 $\mathrm{mg} / \mathrm{kg}$ by gavage immediately after ureteral ligation and then administered daily. As shown in Figure 1a and c, UUO-injured kidneys displayed severe morphologic lesions with tubular dilation and interstitial expansion with collagen accumulation. By contrast, kidneys from animals treated with nintedanib with/without gefitinib exhibited an attenuation of the morphologic lesions with less fibrosis, respectively. Semiquantitative analysis revealed that combined administration of nintedanib and gefitinib reduces Masson-positive areas to a greater extent compared with the treatment with nintedanib or gefitinib alone (Fig. 1a, c). HE staining showed the morphological damage and cell infiltration in the renal tubulointerstitial region after UUO injury, and nintedanib and gefitinib alone or in combination significantly improved this morphological change and reduced cell infiltration (Fig. 1b). This suggests that combined treatment with nintedanib and gefitinib has a synergistic effect to prevent development of renal fibrosis after UUO injury.

\section{Combined Administration of Nintedanib and}

Gefitinib Enforces Inactivation of Renal Interstitial

Fibroblasts and Inhibition of ECM Protein Expression following UUO Injury

Renal fibrosis is characterized by activation of renal interstitial fibroblasts and expression of ECM proteins. Thus, we examined the effect of nintedanib with/without gefitinib on the expression of $\alpha$-SMA, a hallmark of renal interstitial fibroblast activation, and expression of collagen I and fibronectin, 2 ECM proteins, by immunoblot analysis. As shown in Figure $2 a$ and b, coadministration of nintedanib and gefitinib significantly decreased a-SMA, collagen I, and fibronectin protein expression in the UUO-injured kidney to a greater extent than with application of an individual agent alone (Fig. 2a, b).

The metabolism of ECM proteins is regulated by matrix metalloproteinases (MMPs) and tissue inhibitor of metalloproteinases (TIMPs). As shown in Figure $2 \mathrm{c}$ and $\mathrm{d}$, MMP-2 and TIMP-2 expression levels were increased after UUO injury; treatment with either nintedanib or gefitinib alone inhibited TIMP-2 expression, along with increasing MMP-2 expression. The inhibitory effects of these 2 agents in combination on TIMP- 2 were more pronounced than a single agent. In contrast, administration of nintedanib and gefitinib increased expression of MMP-2. Hence, these data demonstrated a synergistic effect of nintedanib and gefitinib to inhibit renal fibroblast activation and ECM protein accumulation, along with promoting ECM degradation in the injured kidney after ureteral obstruction.

\section{Gefitinib Enhances Nintedanib-Elicited Inhibition \\ of Multiple Proinflammatory Cytokine/Chemokine \\ Expression and Macrophage Infiltration in the Kidney after UUO Injury \\ Inflammatory cytokine/chemokine overproduction} and influx of inflammatory cells into renal interstitium is a common pathological feature of renal fibrosis. We thus
Feng/Li/Chao/Huan/Lu/Yi/Jun/Binbin/ $\mathrm{Na} /$ Shougang 


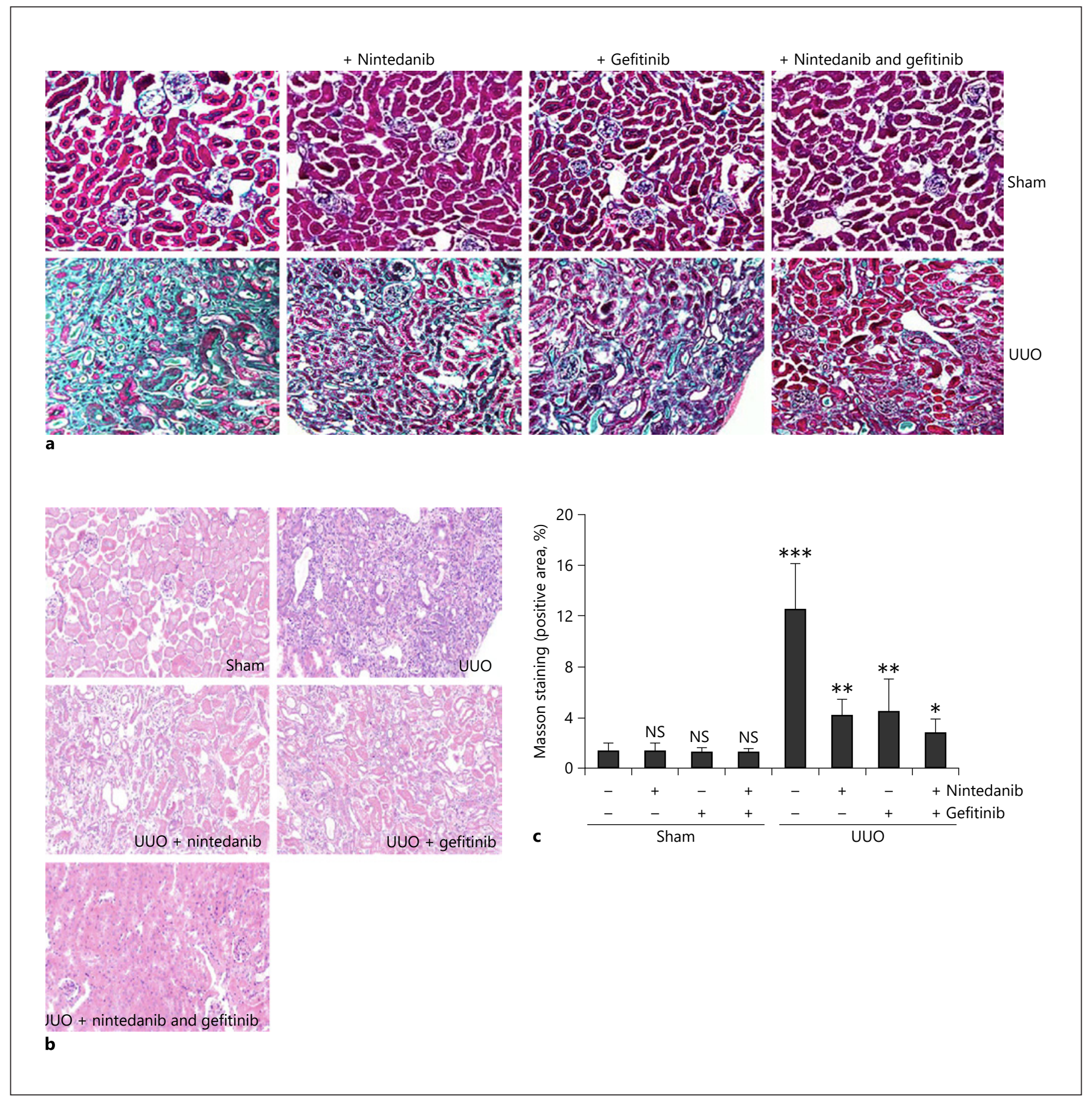

Fig. 1. Nintedanib in combination with gefitinib attenuates renal interstitial fibrosis after UUO injury. a Photomicrographs illustrating Masson staining of kidney tissue with or without nintedanib in combination with gefitinib or not treatment for 7 days $(\times 200)$. b Photomicrographs illustrating HE staining of kidney tissue with or without nintedanib in combination with gefitinib or not treatment for 7 days $(\times 200)$. c The graph shows the percentage of Masson-positive tubulointerstitial area (blue) relative to the whole area from 10 random cortical fields $(\times 200)$ (means \pm SEM) $(n=6) .{ }^{*} p<0.05,{ }^{* *} p<0.01$, and ${ }^{* * *} p<0.001$ versus sham control; NS versus sham control. The number of* means significantly different from one another. UUO, unilateral ureteral obstruction; NS, no significance. 
Fig. 2. Administration of nintedanib in combination with gefitinib reduces expression of a-SMA, collagen I, and fibronectin and modulates expression of MMP-2 and TIMP-2 in the kidney after UUO injury. a Kidneys were taken for immunoblot analysis of $\alpha$-SMA, collagen I, and fibronectin and $\beta$-actin as indicated. Representative immunoblots from 3 experiments are shown. b Expression levels of a-SMA, collagen I, and fibronectin were quantified by densitometry and normalized with $\beta$-actin as indicated. c The kidneys were taken for immunoblot analysis of MMP-2, TIMP-2, and $\beta$-actin as indicated. Representative immunoblots from 3 experiments are shown. d Expression levels of MMP-2 and TIMP-2 were quantified by densitometry and normalized with $\beta$-actin as indicated. Data are represented as the means \pm SEM $(n=6) .{ }^{*} p<0.05,{ }^{* *} p<0.01,{ }^{* * *} p<$ 0.001 , and ${ }^{* * * *} p<0.0001$ versus sham control; NS versus sham control. The number of* means significantly different from one another. UUO, unilateral ureteral obstruction; NS, no significance.

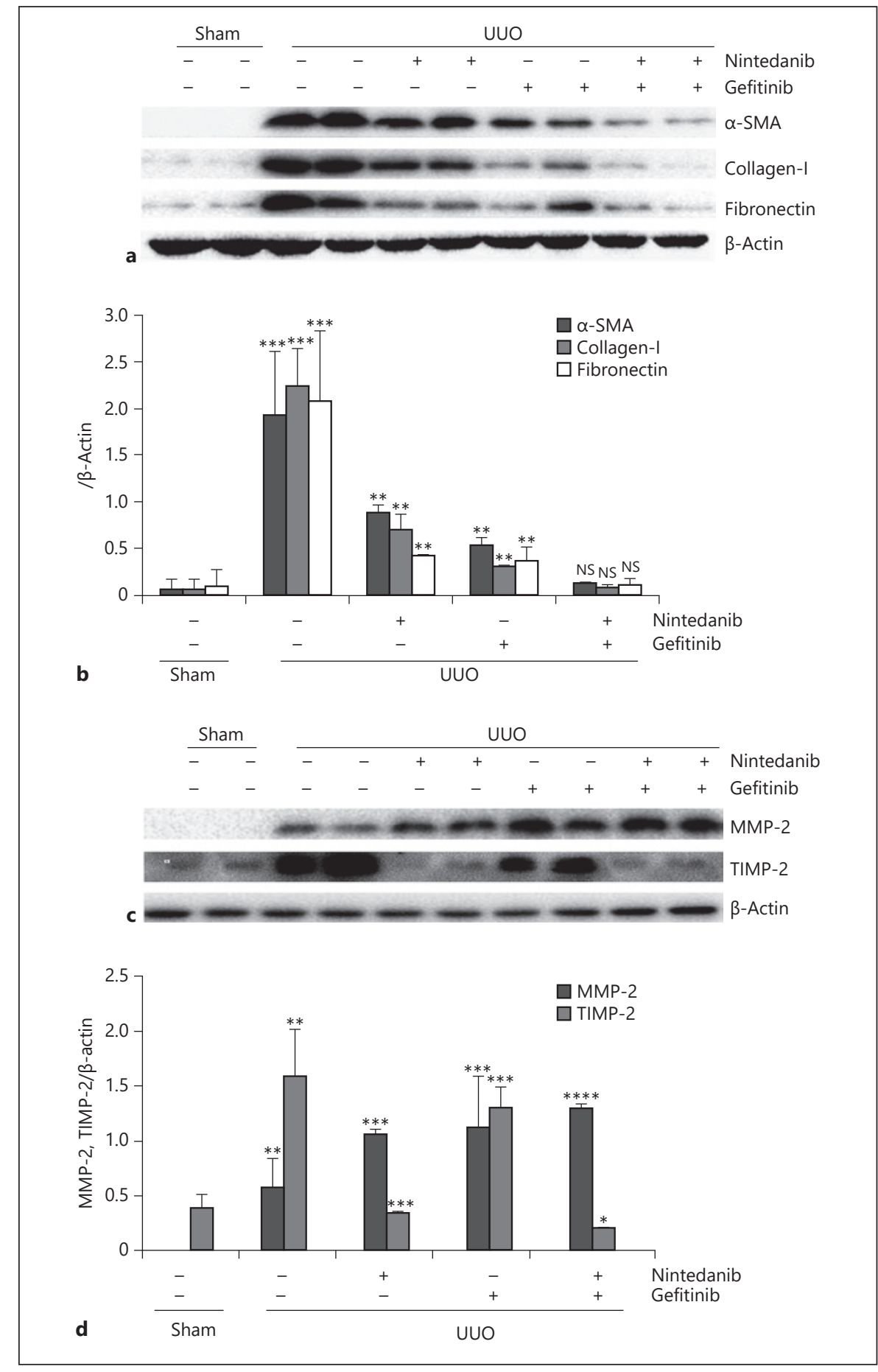

Fig. 3. Nintedanib in combination with gefitinib suppresses the expression of MCP-1, TNF- $\alpha$, IL- $1 \beta$, and IL- 6 and macrophage infiltration in the kidney after UUO injury. a Protein was extracted from the kidneys of mice after UUO injury with or without nintedanib administration, in combination with gefitinib or not, and subjected to the ELISA assay for MCP-1 (a), TNF- $\alpha$ (b), IL-1 $\beta$ (c), and IL-6 (d). e Photomicrographs illustrating immunohistochemical staining of CD68-positive cells in the kidney tissue treat- ed with or without nintedanib, in combination with gefitinib or not for 7 days. $f$ The graph shows the percentage of immunohistochemistry-positive area relative to the whole area from 10 random cortical fields $(\times 200)$. Data are represented as the means \pm SEM $(n$ $=6) .{ }^{*} p<0.05,{ }^{* *} p<0.01$, and ${ }^{* * *} p<0.001$ versus sham control. The number of* means significantly different from one another. UUO, unilateral ureteral obstruction.

(For figure see next page.)

38
Feng/Li/Chao/Huan/Lu/Yi/Jun/Binbin/ $\mathrm{Na} /$ Shougang 

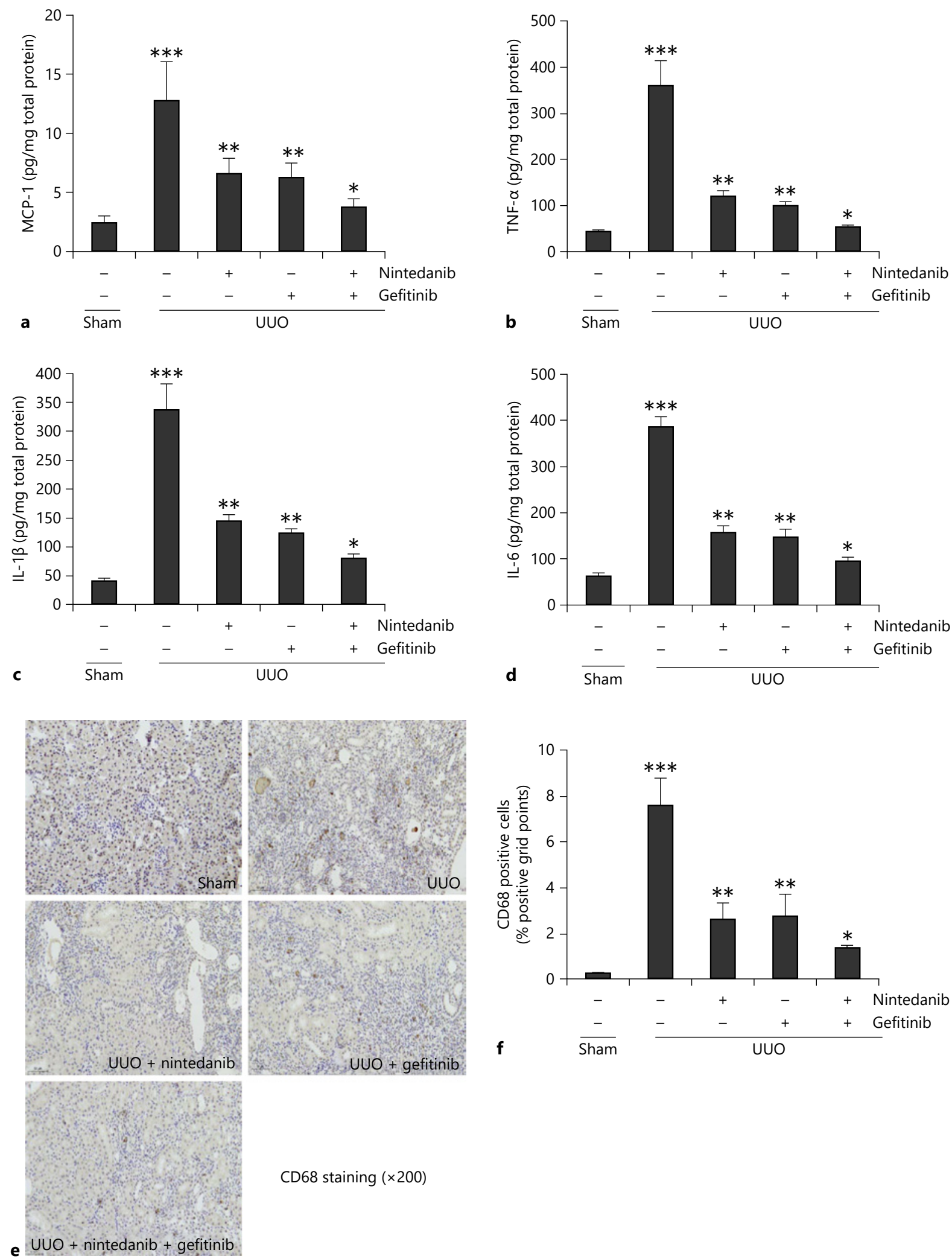
examined the effect of nintedanib with/without gefitinib on the expression of multiple inflammatory cytokines/ chemokines, including monocyte chemoattractant protein-1 (MCP-1), tumor necrosis factor- $\alpha$ (TNF- $\alpha$ ), interleukin-1 $\beta$ (IL-1 $\beta$ ), and interleukin-6 (IL-6) after UUO injury by ELISA. As shown in Figure 3a-d, administration of nintedanib and/or gefitinib significantly reduced increased expression of these cytokines in the kidney after UUO injury. Combined treatment with nintedanib and gefitinib was more potent in inhibiting expression of those cytokines and chemokines than administration of individual agents.

We also examined the effect of nintedanib and/or gefitinib on the macrophage infiltration by immunohistochemical staining using an antibody against CD68 and F4/80, 2 key markers of active macrophages [15]. As shown in Figure $3 \mathrm{e}$ and $\mathrm{f}$ and Figure $4 \mathrm{a}$ and $\mathrm{b}$, the number of CD68-positive or F4/80-positive macrophages were increased in the injured kidney compared with sham-operated kidneys; administration of nintedanib or gefitinib significantly reduced macrophage infiltration. The inhibitory effects of the combination of these 2 agents were more pronounced than single-agent administration. The same results were also demonstrated by immunofluorescent staining on F4/80 (Fig. 4c). These data indicated that combination of nintedanib with gefitinib also has a synergistic effect in suppressing inflammatory responses in the kidney after obstruction.

\section{Combined Administration of Nintedanib and}

Gefitinib Has a Synergistic Effect in Inhibiting Src

and EGFR Phosphorylation in the Kidney after

Obstruction

Since multiple RTKs such as PDGFR $\beta$, FGFR1, and VEGFR2 are the targets of nintedanib, we examined the effect on their phosphorylation in the kidney at day 7 after UUO injury. As shown in Figure 5, administration of nintedanib immediately after obstruction resulted in a significant reduction in PDGFR $\beta$, FGFR1, and VEGFR2 phosphorylation. However, the phosphorylation of these receptors was not affected by gefitinib. Src, one of nRTKs, is also the target of nintedanib. As expected, nintedanib treatment reduced phosphorylation of Src, while gefitinib did not affect Src phosphorylation at the concentration that reduced EGFR phosphorylation in the UUO kidney. Interestingly, nintedanib also showed an inhibitory effect on EGFR phosphorylation, whereas expression of total Src and EGFR protein was not altered by nintedanib and/ or gefitinib (Fig. 6). Therefore, nintedanib in combination with gefitinib has a synergistic effect in blocking Src and EGFR phosphorylation in the kidney after obstruction. Furthermore, nintedanib has a small inhibitory effect on EGFR phosphorylation.

\section{Combined Administration of Nintedanib and}

Gefitinib Has a Synergistic Effect in Inhibiting Smad3,

STAT3, and NF- $\kappa B$ Phosphorylation in the Kidney

after Obstruction

Activation of Smad3, STAT3, and NF-kB signaling pathways is associated with renal fibrosis and subjected to regulation by many RTKs. As shown in Figure 7, treatment with either nintedanib or gefitinib reduced phosphorylation of UUO-induced STAT3 and NF$\kappa B p 65$. Combined treatment with both nintedanib and gefitinib resulted in a greater inhibition on the phosphorylation of all these molecules relative to administration of either of those inhibitors. These data suggest that administration of nintedanib in combination with gefitinib is more potent in inhibiting phosphorylation of Smad3, STAT3, and NF- $\kappa$ B in the kidney after obstruction.

Synergistic Effect of Nintedanib and Gefitinib in

Reducing TGF- $\beta 1$-Induced Activation of Renal

Interstitial Fibroblasts and Production of ECM in

Cultured Renal Interstitial Fibroblasts

We further examined the effect of nintedanib and/or gefitinib on TGF- $\beta 1$-induced activation of renal interstitial fibroblasts in vitro. NRK-49F cells were pretreated with TGF- $\beta 1(5 \mathrm{ng} / \mathrm{mL})$ for $24 \mathrm{~h}$ and then exposed to nintedanib (400 nM) and gefitinib (10 nM) for an additional $48 \mathrm{~h}$ in the continuous presence of TGF- $\beta 1$ ( $5 \mathrm{ng} /$ $\mathrm{mL}$ ) (Fig. 8). Our results demonstrated that TGF- $\beta 1$ induced the expression of $\alpha$-SMA, collagen I, and fibronectin, but that nintedanib or gefitinib inhibited their expression (Fig. 8b-d). Furthermore, nintedanib in combination with gefitinib reduced expression of these 3 proteins to the basal levels (Fig. 8). These data indicate that nintedanib in combination with gefitinib has the synergistic effect in blocking TGF- $\beta 1$-induced activation of renal interstitial fibroblasts and production of ECM proteins.

Synergistic Effect of Nintedanib and Gefitinib in Inhibiting TGF- $\beta 1$-Induced Phosphorylation of Akt,

STAT3, and Smad3 in Cultured Renal Interstitial Fibroblasts

To confirm the effect of nintedanib and/or gefitinib on the activation of profibrotic signaling pathways in cultured NRK-49F cells, we examined the phosphoryla-
Feng/Li/Chao/Huan/Lu/Yi/Jun/Binbin/ $\mathrm{Na} /$ Shougang 
Fig. 4. Nintedanib in combination with gefitinib suppresses the F4/80-positive macrophage infiltration in the kidney after UUO injury. a Photomicrographs illustrating immunohistochemical staining of F4/80-positive cells in the kidney tissue treated with or without nintedanib, in combination with gefitinib or not for 7 days. $\mathbf{b}$ The graph shows the percentage of immunohistochemistry-positive area relative to the whole area from 10 random cortical fields $(\times 200)$. c Photomicrographs illustrating immunofluorescent staining of F4/80-positive cells in the kidney tissue treated with or without nintedanib, in combination with gefitinib or not for 7 days. Data are represented as the means \pm SEM $(n=6) .{ }^{*} p<0.05,{ }^{* *} p<0.01$, and *** $p<0.001$ versus sham control. The number of $*$ means significantly different from one another. UUO, unilateral ureteral obstruction.

Nintedanib and Gefitinib Inhibit Fibrosis

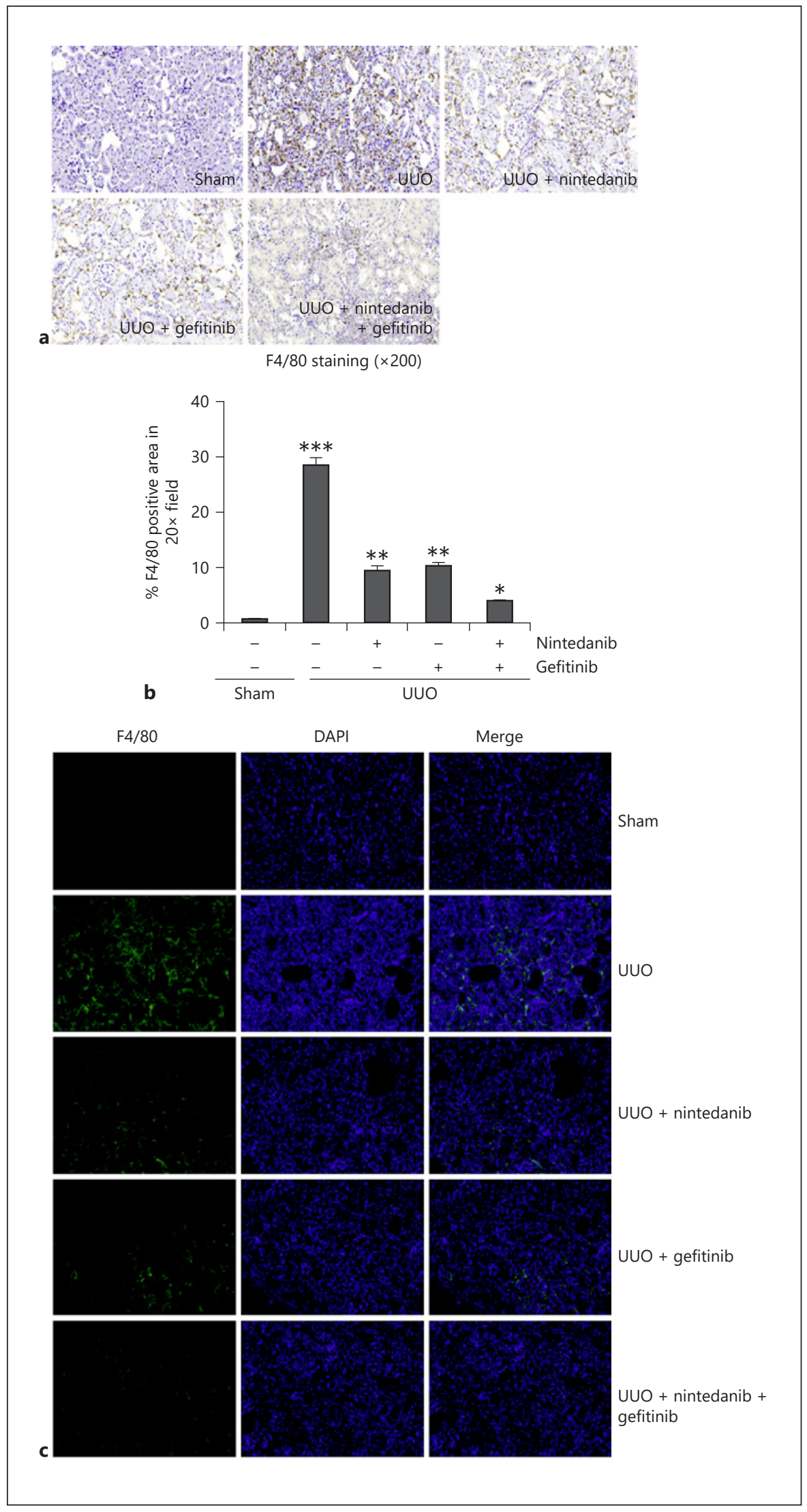

Kidney Dis 2021;7:34-49 


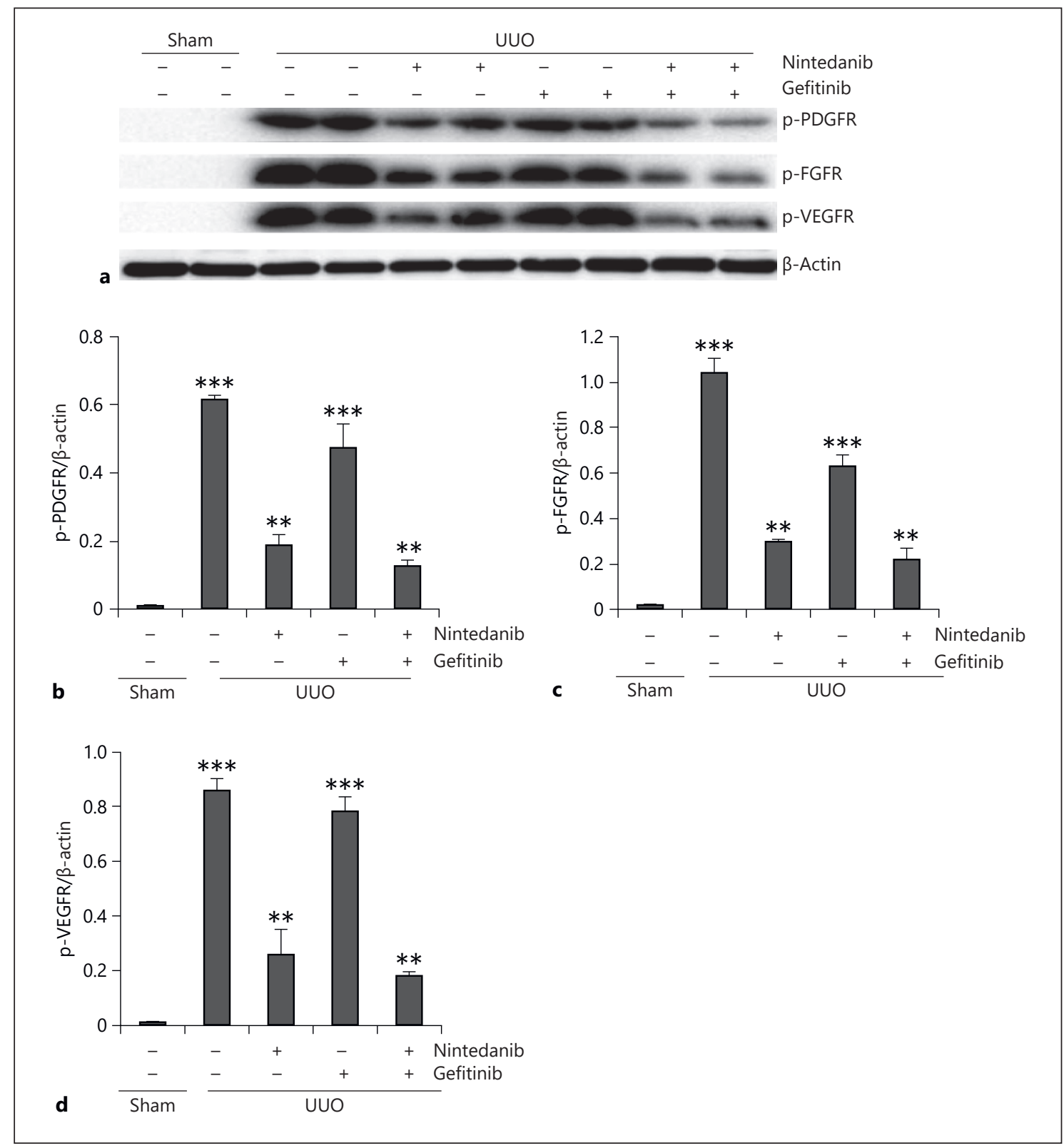

Fig. 5. Effect of nintedanib in combination with gefitinib on UUOinduced activation of PDGFR $\beta$, FGFR1, and VEGFR2 in the kidney. a The kidneys were taken for immunoblot analysis of p-PDGFR $\beta$, p-FGFR1, p-VEGFR2, and $\beta$-actin as indicated. Representative immunoblots from 3 experiments are shown. Expression levels of p-PDGFR $\beta$ (b), p-FGFR1 (c), and p-VEGFR2 (d)

tion and expression of Akt, STAT3, and Smad3 by immunoblot analysis. As shown in Figure 9, TGF- $\beta 1$ induced phosphorylation of Akt, STAT3, and Smad3, while nintedanib or gefitinib treatment significantly reduced their phosphorylation without influencing their total expression levels (Fig. 9b). The combination of nintedanib were quantified by densitometry and normalized with $\beta$-actin as indicated. Data are represented as the means \pm SEM $(n=6)$. ${ }^{* *} p<0.01$ and ${ }^{* * *} p<0.001$ versus sham control. The number of * means significantly different from one another. UUO, unilateral ureteral obstruction; p-PDGFR $\beta$, phospho-PDGFR $\beta$; p-FGFR1, phospho-FGFR1; p-VEGFR2, phospho-VEGFR2.

with gefitinib further reduced phosphorylation of these signaling molecules. This suggests that combination therapy with these 2 inhibitors is more effective than either of them in suppressing these profibrotic signaling pathways. 


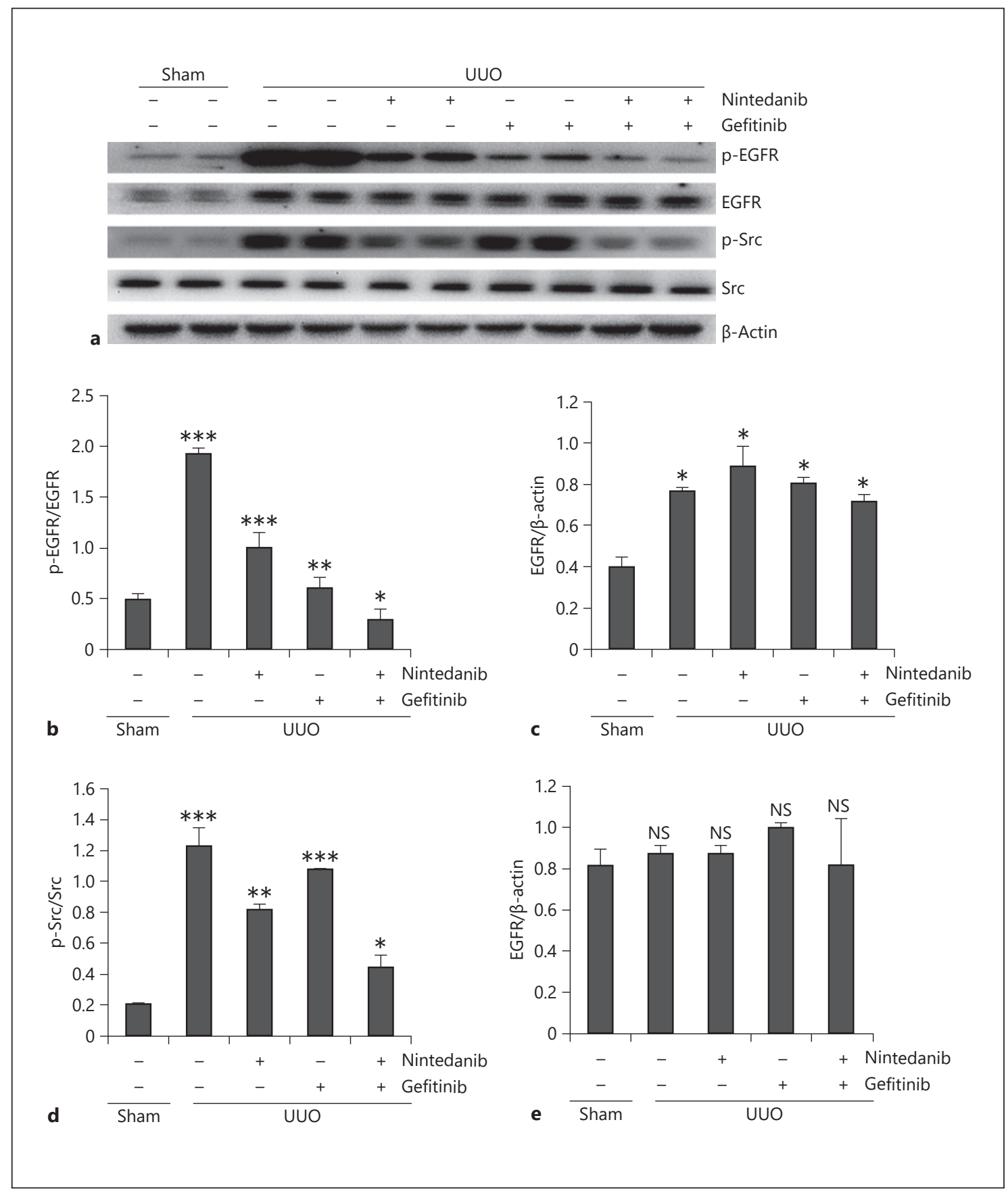

Fig. 6. Effect of nintedanib in combination with gefitinib on UUOinduced activation of EGFR and Src in the kidney. a The kidneys were taken for immunoblot analysis of p-EGFR, p-Src, and total EGFR and Src as indicated. Representative immunoblots from 3 experiments are shown. Expression levels of p-EGFR (b) and p-Src (d) were quantified by densitometry and normalized with total EGFR and Src as indicated, respectively. Expression levels of total EGFR (c) and $\operatorname{Src}(\mathbf{e})$ were quantified by densitometry and normalized with $\beta$-actin as indicated, respectively. Data are represented as the means $\pm \operatorname{SEM}(n=6) .{ }^{*} p<0.05,{ }^{* *} p<0.01$, and ${ }^{* * *} p<0.001$ versus sham control. NS versus sham control. The number of $*$ means significantly different from one another. UUO, unilateral ureteral obstruction; EGFR, epidermal growth factor receptor; p-EGFR, phosphoEGFR; p-Src, phospho-Src; NS, no significance. 


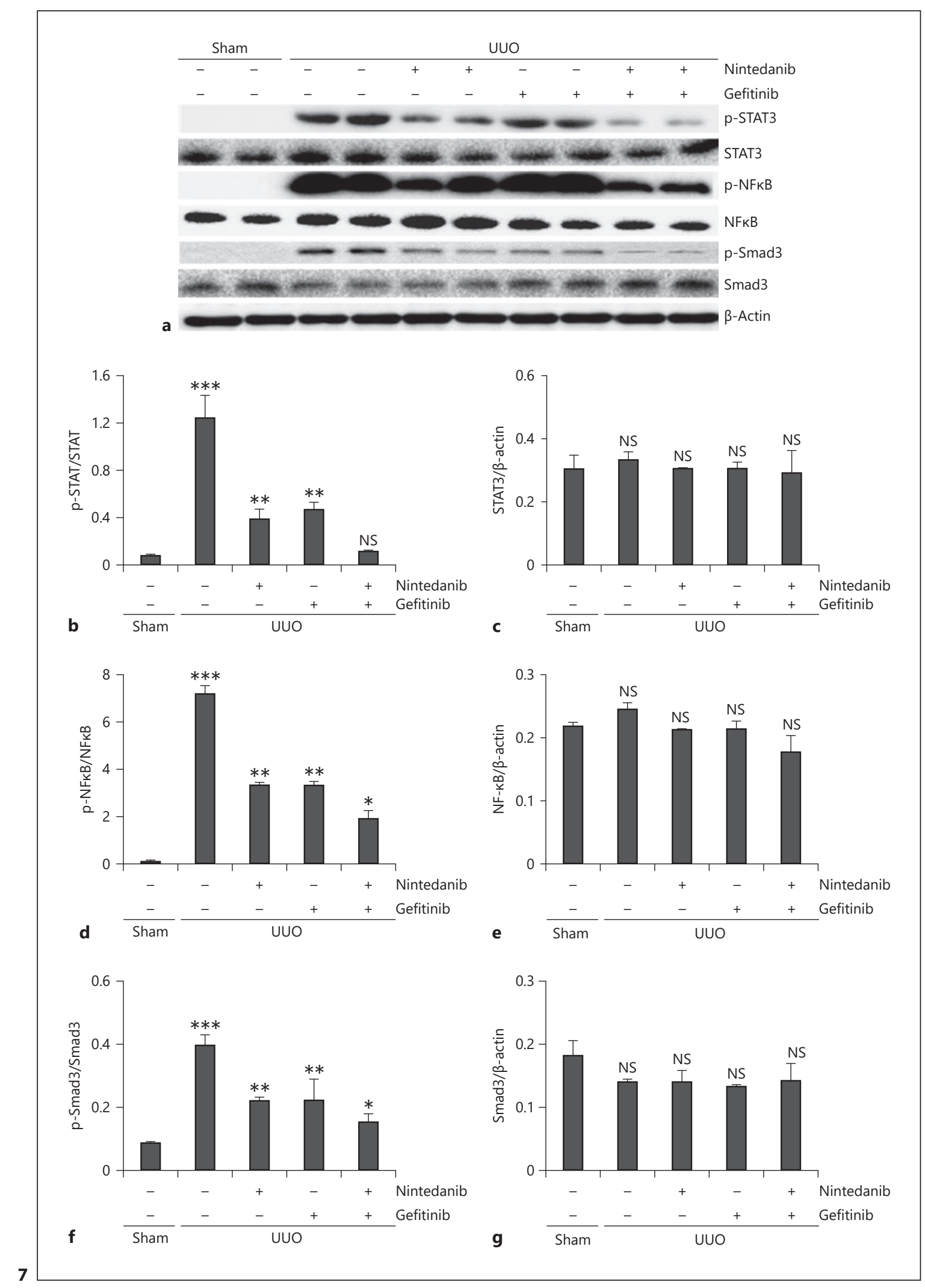

(For legend see next page.) 


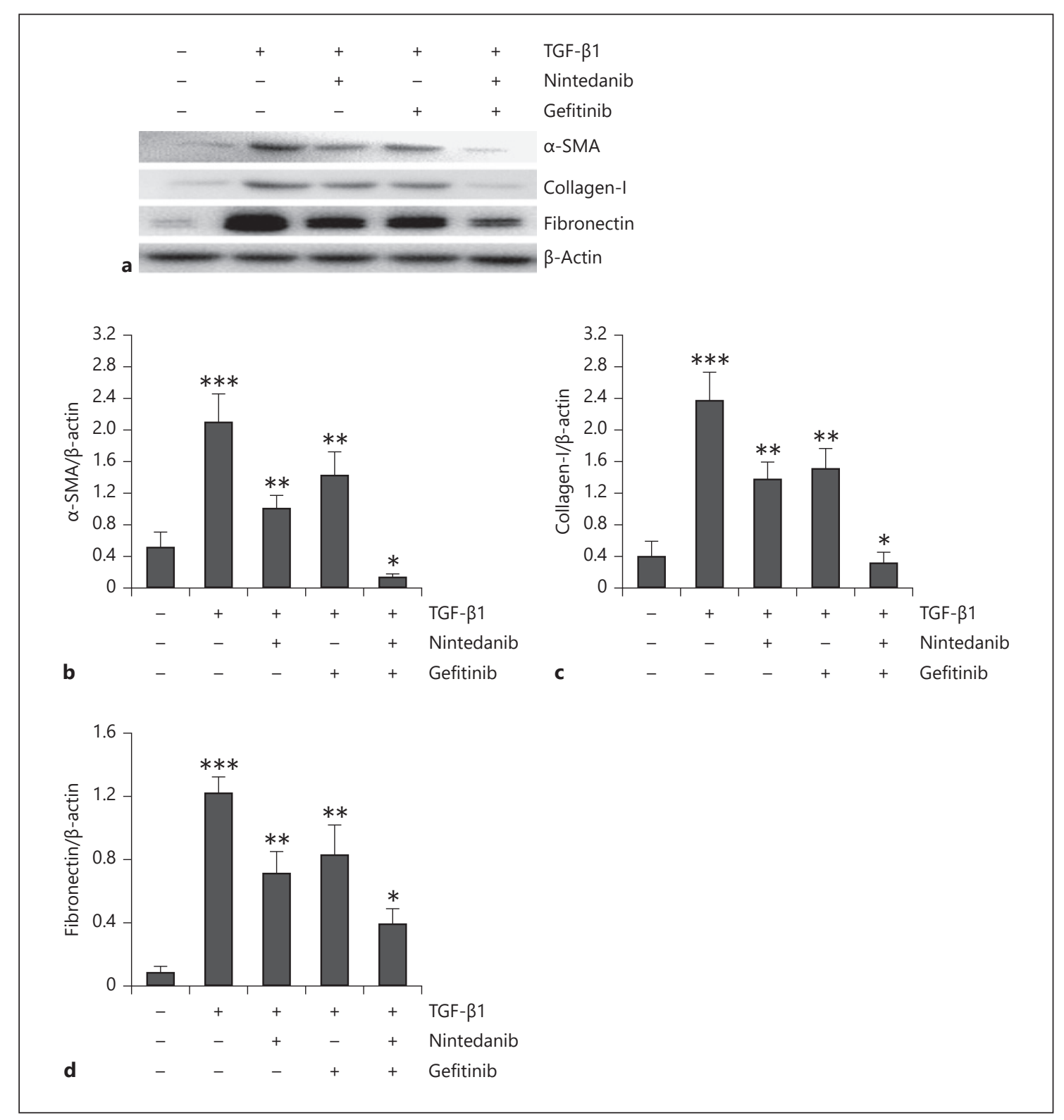

Fig. 8. Nintedanib in combination with gefitinib inhibits TGF- $\beta 1$ induced activation of renal interstitial fibroblasts and production of ECM. a Immunoblot analysis shows the levels of a-SMA, collagen I, and fibronectin protein in NRK-49F cells after treatment by TGF- $\beta 1$ in the presence or absence of nintedanib, in combination with gefitinib or not. Expression levels of $\alpha$-SMA (b), type I

Fig. 7. Nintedanib in combination with gefitinib inhibits UUOinduced activation of STAT3, NF- $\kappa$ B, and Smad3 in the kidney. a Kidneys were taken for immunoblot analysis of p-STAT3, p-NF-

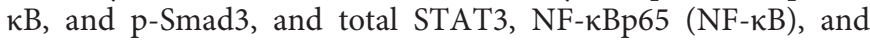
$\mathrm{Smad} 3$ as indicated. Representative immunoblots from 3 experiments are shown. Expression levels of p-STAT3 (b), p-NF- $\kappa$ Bp65 (d), and $\mathrm{p}$-Smad3 (f) were quantified by densitometry and normalized with total STAT3, NF- $\mathrm{\kappa Bp} 65$, and Smad3 as indicated, respec- collagen (c), and fibronectin (d) were quantified by densitometry and normalized with $\beta$-actin. Data are represented as the means \pm SEM $(n=6) .{ }^{*} p<0.05,{ }^{* *} p<0.01$, and ${ }^{* * *} p<0.001$ versus sham control. The number of $*$ means significantly different from one another. ECM, extracellular matrix.

tively. Expression levels of total STAT3 (c), NF-кBp65 (e), and Smad3 (g) were quantified by densitometry and normalized with $\beta$-actin as indicated, respectively. Data are represented as the means $\pm \operatorname{SEM}(n=6) .{ }^{*} p<0.05,{ }^{* *} p<0.01$, and ${ }^{* * *} p<0.001$ versus sham control. NS versus sham control. The number of* means significantly different from one another. UUO, unilateral ureteral obstruction; p-STAT3, phospho-STAT3; p-NF- $\kappa$ B, phospho-NFкBp65; p-Smad3, phospho-Smad3. 
Fig. 9. Nintedanib in combination with gefitinib inhibits TGF- $\beta 1$-induced activation of Akt, Smad3, and STAT3 in cultured renal interstitial fibroblasts. a Immunoblot analysis shows the levels of p-Akt, pSmad3, and p-STAT3, and total Akt, Smad3, and STAT3 as indicated. Representative immunoblots from 3 experiments are shown. $\mathbf{b}$ Expression levels of p-Akt, pSmad3, and p-STAT3 were quantified by densitometry and normalized with total Akt, Smad3, and STAT3 as indicated, respectively. Data are represented as the means $\pm \operatorname{SEM}(n=6) .{ }^{*} p<0.05,{ }^{* *} p<0.01$, and ${ }^{* * *} p<0.001$ versus sham control. The number of $*$ means significantly different from one another. p-Akt, phospho-Akt; pSmad3, phospho-Smad3; p-STAT3, phospho-STAT3.

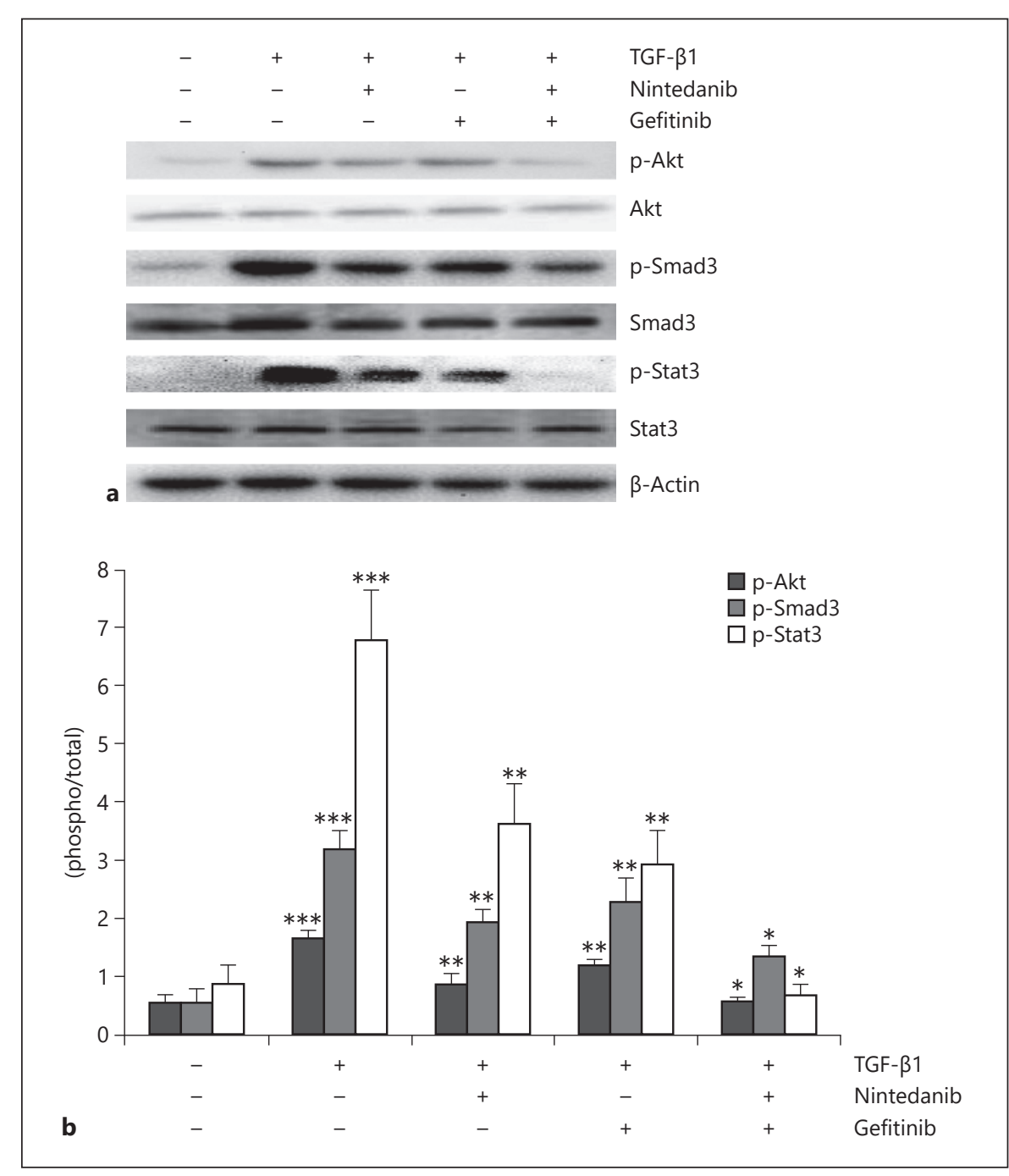

\section{Discussion}

Nintedanib, a potent, indolinone-derived small molecule and multiple-receptor TKI, has been shown to inhibit fibrosis in several organs, including the lung [16, 17], liver [6], kidney [12], and skin [7] and approved by the FDA for the treatment of patients with idiopathic pulmonary fibrosis (IPF) [11]. Our previous studies have also indicated that gefitinib, a specific inhibitor of EGFR, inhibited renal fibrosis and peritoneal fibrosis in animal models $[13,18]$. In the current study, we provide evidence that combined administration of nintedanib and gefitinib exhibits a more potent effect than either of them alone in attenuating development of renal fibrosis, as indicated by reduced renal interstitial fibroblast activation, decreased ECM deposition, and suppressed proinflam- matory cytokine expression and macrophage infiltration. Similarly, we observed a synergistic effect of nintedanib and gefitinib in inhibiting TGF- $\beta 1$-induced activation of renal fibroblasts and ECM protein expression in vitro. On this basis, we suggest that combined administration of nintedanib and gefitinib would be a more effective strategy than monotherapy for attenuation of renal fibrosis.

It has been identified that multiple factors and signaling pathways are associated with renal fibrogenesis [1]. Unfortunately, successful translation of antifibrotic treatments from animals to humans has been limited due to the less effect when blocking a single profibrotic mediator or pathway. This suggests that blockage of multiple fibrotic factors simultaneously may be more effective in alleviating or even reversing fibrosis [19]. Our in vivo 
data here confirmed the antifibrotic effect of nintedanib and gefitinib in renal fibrosis when they were used individually and demonstrated a more potent antifibrotic effect of these 2 inhibitors when they were administered in combination. As expected, the antifibrotic effect of nintedanib and gefitinib is associated with their targeting inhibition of PDGFR $\beta$, FGFR1, VEGFR2, and Src as well as EGFR, respectively. To our surprise, administration of nintedanib alone also showed a significant inhibition on EGFR phosphorylation while nintedanib in combination with gefitinib exhibited an even greater effect on EGFR phosphorylation. These data suggest that nintedanib is able to not only block activation of previously recognized multiple RTKs (i.e., PDGFR $\beta$, FGFR1, and VEGFR2) and Src but also inhibit EGFR activation.

The mechanism by which nintedanib suppresses EGFR is unclear. One possibility is that a cross talk exits between EGFR and other nintedanib-targeted receptors. In this regard, a recent report indicated that nintedanib can inhibit EGFR expression and phosphorylation of AKT in lung fibroblasts derived from patients with idiopathic pulmonary fibrosis [20]. However, this may not be the case in fibrotic kidney since our data showed that nintedanib treatment suppressed EGFR phosphorylation but did not affect expression of total EGFR. Another possibility is that nintedanib may inhibit EGFR phosphorylation through an Src-mediated mechanism. Previous studies have shown that EGFR is activated not only by its own ligands but also by other substances that induce activation of RTKs and G-protein-coupled receptors such as endothelin through a mechanism named transactivation. In this regard, Src can directly induce EGFR phosphorylation at $\mathrm{Tyr}^{845}$ or indirectly by promoting EGFR ligand release via activation of proteases at the cellular membrane [21]. If this is the case, nintedanib would be able to suppress EGFR activation through Src. This hypothesis was supported by our observations that nintedanib-elicited inhibition of EGFR is coincident with reducing Src phosphorylation levels. Nevertheless, as indicated in our study, gefitinib is more potent than nintedanib in inhibiting EGFR and their combined effect was even more potent than either of them alone. Therefore, a combination therapy with the 2 inhibitors is expected to gain a more powerful antifibrotic effect in chronic fibrotic kidney diseases.

Reduction in matrix accumulation is key to attenuate renal fibrosis for an antifibrotic therapy. Matrix accumulation is a consequence of imbalance between matrix production and its degradation, a process that is regulated by MMPs and TIMPs [22]. TIMP-2 is the inhibitor of MMP-
2, while MMP-2 promotes ECM degradation. Since nintedanib has been reported to increase the expression of pro-MMP-2 and inhibit the expression of TIMP-2 in fibroblasts isolated from lungs of IPF patients [23], we examined the effect of nintedanib, gefitinib, or their combination on the expression of these 2 molecules. Our results indicated that administration of either nintedanib or gefitinib inhibited TIMP-2 and increased MMP-2 expression, and their combination showed a greater inhibitory effect on the expression of these 2 molecules, suggesting that a combination therapy with these 2 agents would be able to effectively regulate the balance of MMP-2 and TIMP-2 toward promoting degradation of ECM components. Of course, in addition to MMP-2 and TIMP-2, nintedanib and gefitinib may also regulate expression of other MMPs and TIMPs. Further studies are needed to examine the effect of these 2 inhibitors on the expression of other MMPs and TIMPs in the fibrotic kidney.

Inflammation is recognized as a critical driver in the pathogenesis of renal fibrosis [1]. It has been shown that inflammatory pathways regulated MMP expression and that MMPs regulate the expression of inflammatory mediators and subsequent renal injury [22]. Our present data demonstrated that expression of some proinflammatory cytokines/chemokines, such as MCP-1, TNF- $\alpha$, IL-6, and IL- $1 \beta$, was increased and that CD68-positive and F4/80-positive macrophage cells were infiltrated in the kidney after UUO injury. Administration of nintedanib and/or gefitinib significantly inhibited expression of the aforementioned proinflammatory cytokines and infiltration of CD68-positive and F4/80-positive macrophages, and the anti-inflammation effect in the combination group is more pronounced than that in the monotherapy group. Similarly, nintedanib also significantly reduced inflammatory cell infiltration, IL- $1 \beta$ expression, and granuloma formation in experimental models of lung fibrosis $[14,24]$.

The anti-inflammatory effect of nintedanib and/or gefitinib may be through inhibition of some transcription factors. In this context, activation of transcription factors NF$\kappa \mathrm{B}$ and STAT3 has been reported to be necessary for production of multiple cytokines/chemokines and the progression of renal fibrosis [1]. TGF- $\beta 1$ can induce the activation of STAT3 and NF- $\mathrm{BB}$ pathways [25], while STAT3 and NF- $\kappa \mathrm{B}$ are also able to promote expression of TGF- $\beta$ and its receptors $[1,25,26]$. In this study, we demonstrated that treatment with nintedanib and/or gefitinib significantly suppressed phosphorylation of STAT3 and NF- $\kappa \mathrm{B}$ as well as Smad3, a key mediator in TGF- $\beta$ signaling pathway in the UUO-injured kidney. In the cultured renal 
fibroblasts exposed to TGF- $\beta 1$, combined treatment with nintedanib and gefitinib also suppressed activation of renal interstitial fibroblasts and overproduction of ECM proteins to a great extent compared with either agent alone. As such, it appears that the combination of nintedanib with gefitinib could be more effective in reducing transduction of fibrotic signals, inhibiting fibroblast activation, inflammation and ECM synthesis, and promoting ECM degradation.

Clinical trials and clinical application have demonstrated the efficacy of nintedanib in treating IPF patients [27]. More and more preclinical studies have demonstrated the antifibrotic effect of TKIs in a variety of animal models of organ fibrosis, including renal fibrosis [4, 28]. Current preclinical data have verified that combined administration of nintedanib and gefitinib has a stronger antifibrotic effect than a single agent. Although it has been reported that either nintedanib or gefitinib can cause some side effects, such as rash, vomiting, and diarrhea in clinical patients $[29,30]$, we did not observe such adverse effects during 1 -week administration of these 2 agents. A possible explanation is that nintedanib, gefitinib, or their combinations were only used for a short period time in the current study. Other animal models and a longer period of administration of these drugs are needed to further examine the efficacy, tolerability, and side effects of combination of these 2 agents in the treatment or even reversal of fibrotic renal diseases in the future.

\section{Conclusion}

We were for the first time to demonstrate that combined administration of nintedanib and gefitinib achieved better protection from UUO-induced renal fibrosis than single-agent administration. The underlying mechanism is related to inhibition of multiple cellular events associated with renal fibrogenesis, including renal fibroblast activation, ECM synthesis, and inflammation. Therefore, combination of nintedanib and gefitinib may be a novel therapeutic treatment for fibrotic renal diseases.

\section{Acknowledgements}

The authors thank the medical staff at the Department of Nephrology, Shanghai East Hospital, Tongji University School of Medicine, Shanghai, China. They acknowledge the laboratory support provided by the Translational Medicine Platform of Shanghai East Hospital, Tongji University School of Medicine, Shanghai, China.

\section{Statement of Ethics}

The animal experiments conform to internationally accepted standards and have been approved by the Ethics Committee of Tongji University (reference number 81670623).

\section{Conflict of Interest Statement}

The authors have no conflicts of interest to declare.

\section{Funding Sources}

This study was supported by the grants from Science, Technology and Economic Committee of Pudong New District of Shanghai, China (PKJ2017-Y19 to Liu Feng), the Academic Leader Training Plan of Health System of Pudong New District of Shanghai, China (PWRd2019-13 to Liu Feng), the National Nature Science Foundation of China (81670623 and 81830021 to Zhuang Shougang), and the Key Discipline Construction Project of Pudong Health Bureau of Shanghai, China (PWZxk2017-05 to Liu Na).

\section{Author Contributions}

L.F. and Z.S. designed experiments. W.L., Y.C., Q.H., F.L., W.Y., W.J., C.B., and L.N. conducted the experiments. L.F. analyzed data. L.F. and Z.S. prepared the manuscript.

References Annu Rev Physiol. 2018 Feb 10;80:309-26.

2 François H, Chatziantoniou C. Renal fibrosis: recent translational aspects. Matrix Biol. 2018 Aug;68-69:318-32.

3 Klinkhammer BM, Goldschmeding R, Floege J, Boor P. Treatment of renal fibrosis-turning challenges into opportunities. Adv Chronic Kidney Dis. 2017 Mar;24(2):117-29.

4 Liu F, Zhuang S. Role of receptor tyrosine kinase signaling in renal fibrosis. Int J Mol Sci. 2016 Jun 20;17(5):972.

5 Macagno F, Varone F, Leone PM, Mari PV, Panico L, Berardini L, et al. New treatment directions for IPF: current status of ongoing and upcoming clinical trials. Expert Rev Respir Med. 2017 Jul;11(7):533-48.

6 Öztürk Akcora B, Storm G, Prakash J, Bansal R. Tyrosine kinase inhibitor BIBF1120 ameliorates inflammation, angiogenesis and fibrosis in CCl4-induced liver fibrogenesis mouse model. Sci Rep. 2017 Mar 14;7:44545.

7 Huang J, Beyer C, Palumbo-Zerr K, Zhang Y, Ramming A, Distler A, et al. Nintedanib inhibits fibroblast activation and ameliorates fibrosis in preclinical models of systemic sclerosis. Ann Rheum Dis. 2016 May;75(5):883-90.
48

Kidney Dis 2021;7:34-49 DOI: $10.1159 / 000509670$
Feng/Li/Chao/Huan/Lu/Yi/Jun/Binbin/ $\mathrm{Na}$ /Shougang 
8 Huang J, Maier C, Zhang Y, Soare A, Dees C, Beyer C, et al. Nintedanib inhibits macrophage activation and ameliorates vascular and fibrotic manifestations in the Fra2 mouse model of systemic sclerosis. Ann Rheum Dis. 2017 Nov;76(11):1941-8.

9 Woodcock HV, Molyneaux PL, Maher TM. Reducing lung function decline in patients with idiopathic pulmonary fibrosis: potential of nintedanib. Drug Des Devel Ther. 2013;7: 503-10.

10 Richeldi L, du Bois RM, Raghu G, Azuma A, Brown KK, Costabel U, et al. Efficacy and safety of nintedanib in idiopathic pulmonary fibrosis. N Engl J Med. 2014 May 29;370(22): 2071-82.

11 McCormack PL. Nintedanib: first global approval. Drugs. 2015 Jan;75(1):129-39.

12 Liu F, Wang L, Qi H, Wang J, Wang Y, Jiang $\mathrm{W}$, et al. Nintedanib, a triple tyrosine kinase inhibitor, attenuates renal fibrosis in chronic kidney disease. Clin Sci. 2017 Jul;131(16): 2125-43.

13 Liu N, Wang L, Yang T, Xiong C, Xu L, Shi Y, et al. EGF receptor inhibition alleviates hyperuricemic nephropathy. J Am Soc Nephrol. 2015 Nov;26(11):2716-29.

14 Wang L, Liu N, Xiong C, Xu L, Shi Y, Qiu A, et al. Inhibition of EGF receptor blocks the development and progression of peritoneal fibrosis. J Am Soc Nephrol. 2016 Sep;27(9):2631-44.

15 Uchida T, Oda T, Matsubara H, Watanabe A, Takechi H, Oshima N, et al. Renoprotective effects of a dipeptidyl peptidase 4 inhibitor in a mouse model of progressive renal fibrosis. Ren Fail. 2017 Nov;39(1):340-9.
16 Rangarajan S, Kurundkar A, Kurundkar D, Bernard K, Sanders YY, Ding Q, et al. Novel mechanisms for the antifibrotic action of nintedanib. Am J Respir Cell Mol Biol. 2016 Jan;54(1):51-9.

17 Richeldi L, Cottin V, du Bois RM, Selman M, Kimura T, Bailes Z, et al. Nintedanib in patients with idiopathic pulmonary fibrosis: combined evidence from the TOMORROW and INPULSIS ${ }^{\circledR}$ trials. Respir Med. 2016 Apr; 113:74-9.

18 Liu N, Guo JK, Pang M, Tolbert E, Ponnusamy M, Gong R, et al. Genetic or pharmacologic blockade of EGFR inhibits renal fibrosis. J Am Soc Nephrol. 2012 May;23(5):854-67.

19 Schuppan D, Pinzani M. Anti-fibrotic therapy: lost in translation? J Hepatol. 2012; 56(Suppl 1):S66-74.

20 Epstein Shochet G, Brook E, Eyal O, Edelstein E, Shitrit D. Epidermal growth factor receptor paracrine upregulation in idiopathic pulmonary fibrosis fibroblasts is blocked by nintedanib. Am J Physiol Lung Cell Mol Physiol. 2019 Jun 1;316(6):L1025-34.

21 Parsons JT, Parsons SJ. Src family protein tyrosine kinases: cooperating with growth factor and adhesion signaling pathways. Curr Opin Cell Biol. 1997 Apr;9(2):187-92.

22 Parrish AR. Matrix metalloproteinases in kidney disease: role in pathogenesis and potential as a therapeutic target. Prog Mol Biol Transl Sci. 2017;148:31-65.
23 Hostettler KE, Zhong J, Papakonstantinou E, Karakiulakis G, Tamm M, Seidel P, et al. Antifibrotic effects of nintedanib in lung fibroblasts derived from patients with idiopathic pulmonary fibrosis. Respir Res. 2014 Dec 12; 15:157.

24 Wollin L, Maillet I, Quesniaux V, Holweg A, Ryffel B. Antifibrotic and anti-inflammatory activity of the tyrosine kinase inhibitor nintedanib in experimental models of lung fibrosis. J Pharmacol Exp Ther. 2014 May;349(2): 209-20.

25 Meng XM, Tang PM, Li J, Lan HY. TGF- $\beta /$ Smad signaling in renal fibrosis. Front Physiol. 2015;6:82.

26 Liu J, Zhong Y, Liu G, Zhang X, Xiao B, Huang $S$, et al. Role of Stat 3 signaling in control of EMT of tubular epithelial cells during renal fibrosis. Cell Physiol Biochem. 2017; 42(6):2552-8.

27 Aryal S, Nathan SD. An update on emerging drugs for the treatment of idiopathic pulmonary fibrosis. Expert Opin Emerg Drugs. 2018 Jun;23(2):159-72.

28 Korashy HM, Attafi IM, Ansari MA, Assiri MA, Belali OM, Ahmad SF, et al. Molecular mechanisms of cardiotoxicity of gefitinib in vivo and in vitro rat cardiomyocyte: role of apoptosis and oxidative stress. Toxicol Lett. 2016 Jun 11;252:50-61.

29 Cersosimo RJ. Gefitinib: an adverse effects profile. Expert Opin Drug Saf. 2006 May;5(3): 469-79.

30 Rodríguez-Portal JA. Efficacy and safety of nintedanib for the treatment of idiopathic pulmonary fibrosis: an update. Drugs R D. 2018 Mar; 18(1):19-25. 